\title{
8-Node solid-shell elements selective mass scaling for explicit dynamic analysis of layered thin-walled structures
}

\author{
F. Confalonieri · A. Ghisi · U. Perego
}

Received: January 7, 2015 / Accepted: July 25, 2015

\begin{abstract}
To overcome the issue of spurious maximum eigenfrequencies leading to small steps in explicit time integration, a recently proposed selective mass scaling technique, specifically conceived for 8-node hexahedral solid-shell elements, is reconsidered for application to layered shells, where several solid-shell elements are used through the thickness of thin-walled structures.

In this case, the resulting scaled mass matrix is not perfectly diagonal. However, the introduced coupling is shown to be limited to the nodes belonging to the same fiber through the thickness, so that the additional computational burden is almost negligible and by far compensated by the larger size of the critical time step. The proposed numerical tests show that the adopted mass scaling leads to a critical time step size which is determined by the element in-plane dimensions only, independent of the layers number, with negligible accuracy loss, both in small and large displacement problems.
\end{abstract}

Keywords Explicit time integration · selective mass scaling · solid-shell elements · layered thin-walled structures

\section{Introduction}

Solid-shell elements (see, e.g., Hauptmann and Schweizerhof (1998), Tan and VuQuoc (2005), Abed-Meraim and Combescure (2009), Schwarze and Reese (2011), Abed-Meraim et al (2013), Naceur et al (2013)), characterized by displacement degrees of freedom (dofs) only, allow for the implementation of complex, fully three-dimensional constitutive laws. Their use is thus particularly suitable for the analysis of fracture and delamination problems in thin walled structures. However, the element small thickness with respect to the in-plane dimensions makes

Federica Confalonieri, Aldo Ghisi, Umberto Perego

Politecnico di Milano, Department of Civil and Environmental Engineering

Piazza Leonardo da Vinci 32, Milano, Italy

E-mail: federica.confalonieri@polimi.it

E-mail: aldo.ghisi@polimi.it

E-mail: umberto.perego@polimi.it 
the simulation computationally expensive, when a conditionally stable explicit integration scheme is adopted, as it is often done in highly nonlinear problems. In the case of a central difference time integration scheme, the critical time step is determined by the highest eigenfrequency $\omega_{\max }$ of the assembled mesh:

$$
\Delta t \leq \frac{2}{\omega_{\max }}
$$

It can be shown that a conservative bound for the structure critical eigenfrequency can be obtained by considering the maximum eigenfrequency $\omega_{\max }^{e}$ of an individual element of the mesh, i.e.:

$$
\omega_{\max } \leq \max _{e}\left\{\omega_{\max }^{e}\right\} .
$$

Furthermore, the Courant-Friedrichs-Lewy (CFL) condition states that the critical time step coincides with the so called traversal time, i.e. the time required by a dilatational stress wave to run across the shortest element dimension. In the case of solid-shell elements, the CFL condition may lead to extremely low values of the critical time step, since the thickness dimension is, by definition, sensibly smaller than the in-plane ones, thus determining the characteristic length of the element. As a consequence, the computational cost of an explicit simulation can grow significantly when the structure is discretized with solid-shell elements.

Dynamic problems can be generally categorized as either wave propagation or structural dynamics problems. In the first case, the load has a short duration (typically of the order of microseconds) when compared to the to the lowest structural eigenperiods, as in the case of impact or blast loading conditions. The structural response is characterized by short-term transient effects, with stress waves propagating through the structure, and is rich in high frequencies, usually of the order of kilo-Hertz $(\mathrm{kHz})$ or higher. On the other hand, structural dynamics problems are characterized by long dynamic load duration (usually in a time frame from milliseconds to seconds), with a frequency content typically of the order of few hundred Hertz.

According to (Zukas (2004), chapt. 1), there is not clear demarcation between these two areas. He lists a number of indicators to characterize structural dynamic problems. Among these, one of the most important is that structural dynamic problems involve global deformations, primarily caused by the lowest structural eigenmodes. Another usual way to classify dynamic problems is on the basis of the strain rate magnitude. According to Zukas (2004), typical strain rates for structural dynamic problems are in the range of $10^{-2} \div 10^{2}$ per second. Accordig to Meyers (1994), strain rates up to $10^{\circ}$ per second can be classified as "quasistatic", whereas strain rates in the range $10^{1}-10^{3}$ can be classified as "dynamic -low" and fall in the category of structural dynamic problems.

In structural dynamic problems, where the main contribution to the overall dynamical behaviour derives from the lowest eigenfrequencies, related to the rigid body motions of individual elements, a selective mass scaling technique, i.e. a scaling limited to a certain portion of the dofs without affecting the dynamical response, can be introduced to increase the critical time step, in order to reduce the computational burden of the simulation. The basic idea is to modify the solid-shell element mass matrix, artificially scaling down the highest structural eigenfrequencies, without significantly altering the lowest ones. A mass scaling rigorously satisfying this requirement can be obtained by summing to the mass matrix 
the stiffness matrix multiplied by a scaling parameter (Macek and Aubert (1995), Olovsson et al (2005)). This solution does not alter the lowest eigenfrequencies, but it is computationally burdensome, since the diagonal structure of the mass matrix is lost. Moreover, when the problem is highly non linear, for example in the presence of large deformations, the stiffness matrix can change significantly during the analysis. As an alternative to the stiffness matrix, Olovsson et al (2005) proposed to use a fixed added mass matrix, with a kernel including the translational rigid body modes of an individual element. Other techniques for selective mass scaling have been developed in recent years, such as: bipenalty methods limiting both stiffness and mass matrices in Hetherington et al (2012), micro-inertia or inertia penalty formulations both of which can be actually seen, along with the classical mass scaling, in a unified framework, as shown in Askes et al (2011). Tkachuk and Bischoff (2013b) proposed a variational framework for a rigorous approach to the selective mass scaling problem, formulating a penalized mixed Hamilton's principle, based on displacement, velocity and momentum as independent fields in the functional. Other possibilities for the choice of the scaled mass matrix are also explored in Tkachuk and Bischoff (2013a), where optimality criteria are studied such as eigenmode preservation, conditioning and sparsity of the mass matrix.

Most of the cited methods, however, produce non-diagonal scaled mass matrices which require to be inverted at each time step for the computation of the nodal accelerations. A possible solution to this problem has been proposed in Tkachuk and Bischoff (2015), where a method for a direct variational construction of a sparse inverse matrix has been formulated, together with a new selective mass scaling to be applied directly to the inverse mass matrix. A different approach has been considered in Olovsson et al (2004) and Cocchetti et al (2013), where the relative motion in the thickness direction of thin-walled structures has been penalized by the addition of artificial inertia. The method proposed in Cocchetti et al (2013), specifically conceived for parallelepiped solid-shell elements, has been extended to distorted solid-shell elements in Cocchetti et al (2015), together with a strategy for the computation of the optimal scaling factor and of the critical time step size.

In the present work, the selective mass scaling procedure proposed in Cocchetti et al (2015) for single-layer 8-node solid-shell elements is generalized to the case of multi-layer shells. The goal is to penalize the relative motion between the shell upper and lower surfaces, so that the critical time step is determined only by the minimum in-plane size of the elements, as with standard four-nodes shell element meshes, independent of the number of layers or solid-shell elements used for through-the-thickness discretization.

The paper is structured as follows. In section 2 , the selective mass scaling procedure for single-layer shells is briefly described, while the extension to multi-layer shell structures is presented in section 3. The critical issue of the determination of the optimal mass scaling factor and of the computation of the critical time step size is briefly recalled in section 4 , before discussing the numerical examples showing the advantages of the proposed method in section 5. We draw our conclusions in section 6 . 


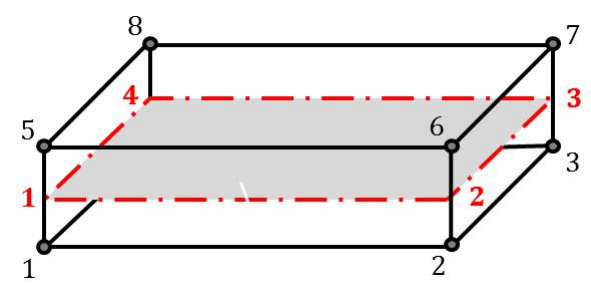

Fig. 1 Eight-node solid-shell element

\section{Selective mass scaling of single layer shells}

The geometry of the reference 8-node solid-shell element is shown in Fig. 1. It consists of a solid 8-node brick element with a dimension, the thickness, that is significantly smaller than the other two, so that it is always possible to identify the element upper and lower surfaces in an unambiguous way. Let $\mathbf{x}=\mathbf{x}(\xi, \eta, \zeta)$ be the isoparametric mapping defining the coordinates $\mathbf{x}$ of a point of the element in terms of the coordinates $\xi, \eta, \zeta$ of a point of its $2 \times 2 \times 2$ parent cube and let $\mathbf{X}$ be the column matrix gathering its nodal coordinates:

$$
\underset{24 \times 1}{\mathbf{X}}=\left[\begin{array}{c}
\mathbf{X}_{1-4} \\
12 \times 1 \\
\mathbf{X}_{5-8} \\
12 \times 1
\end{array}\right],
$$

where $\mathbf{X}_{1-4}$ and $\mathbf{X}_{5-8}$ contain the coordinates of nodes belonging to the element lower and upper surfaces, respectively. Following Cocchetti et al (2013, 2015), the element geometry and kinematics are expressed in terms of variables related to middle surface nodes, as in classical shell elements, and to the element corner fibers, i.e. to the segments connecting corresponding pairs of nodes belonging to the lower and upper surfaces (Fig. 1). The coordinates of the nodes belonging to the middle surface, which will be hereafter referred to as "corner nodes", in grey in Fig. 1, can be defined as:

$$
\underset{12 \times 1}{\mathbf{X}^{m}}=\frac{\mathbf{X}_{5-8}+\mathbf{X}_{1-4}}{2}
$$

The term "corner fiber coordinates" will be used to denote the semi-length $\Delta \mathbf{X}$ of corner fibers:

$$
\underset{12 \times 1}{\Delta \mathbf{X}}=\frac{\mathbf{X}_{5-8}-\mathbf{X}_{1-4}}{2}
$$
$\widehat{\mathbf{X}}$ :

The transformed coordinates $\mathbf{X}^{m}$ and $\Delta \mathbf{X}$ are gathered in the column matrix

$$
\underset{24 \times 1}{\widehat{\mathbf{X}}}=\left[\begin{array}{l}
\mathbf{X}^{m} \\
\Delta \mathbf{X}
\end{array}\right]
$$

A linear transformation can be defined to map the original nodal coordinates $\mathbf{X}$ into the transformed ones $\widehat{\mathbf{X}}$ :

$$
\underset{24 \times 1}{\mathbf{X}}=\underset{24 \times 24}{\mathbf{Q}} \underset{24 \times 1}{\widehat{\mathbf{X}}},
$$


with:

$$
\mathbf{Q}=\left[\begin{array}{cc}
\mathbf{I}_{12} & -\mathbf{I}_{12} \\
\mathbf{I}_{12} & \mathbf{I}_{12}
\end{array}\right], \quad \mathbf{Q}^{-1}=\frac{1}{2} \mathbf{Q}^{T}
$$

where $\mathbf{I}_{12}$ is the $12 \times 12$ identity matrix.

Adopting the same notation introduced for the nodal coordinates, the kinematic solution can be expressed either in terms of the classical nodal dofs or in terms of corner nodes (i.e. nodes belonging to the middle surface) and corner fibers quantities.

Let us consider the element nodal accelerations (the notation extends straightforwardly also to velocities and displacements). As in (3) and (6), the element nodal accelerations vectors $\mathbf{a}_{e}$ and $\widehat{\mathbf{a}}_{e}$, can be defined as:

$$
\mathbf{a}_{e}=\left[\begin{array}{c}
\mathbf{a}_{1-4} \\
\mathbf{a}_{5-8}
\end{array}\right], \quad \widehat{\mathbf{a}}_{e}=\left[\begin{array}{c}
\mathbf{a}^{m} \\
\Delta \mathbf{a}
\end{array}\right],
$$

where:

$$
\mathbf{a}_{e}^{m}=\frac{\mathbf{a}_{5-8}+\mathbf{a}_{1-4}}{2}, \quad \Delta \mathbf{a}_{e}=\frac{\mathbf{a}_{5-8}-\mathbf{a}_{1-4}}{2}
$$

and

$$
\mathbf{a}_{e}=\mathbf{Q} \widehat{\mathbf{a}}_{e} .
$$

The balance momentum equation of an undamped element can be written by using the principle of virtual work as:

$$
\delta \mathbf{a}_{e}^{T} \mathbf{M}_{e} \mathbf{a}_{e}=\delta \mathbf{a}_{e}^{T} \mathbf{f}_{e}
$$

$\delta \mathbf{a}_{e}$ being the virtual accelerations at the nodes of element $e, \mathbf{M}_{e}$ the element mass matrix and $\mathbf{f}_{e}=\mathbf{f}_{e}^{e x t}-\mathbf{f}_{e}^{\text {int }}$ the difference between the vectors of external and internal equivalent nodal forces. A lumped mass matrix is here considered:

$$
\underset{24 \times 24}{\mathbf{M}_{e}}=\left[\begin{array}{cc}
\mathbf{m}_{12 \times 12}^{\text {low }} & \mathbf{0} \\
\mathbf{0} & \mathbf{m}_{12 \times 12}^{u p}
\end{array}\right]
$$

being $\mathbf{m}^{\text {low }}$ and $\mathbf{m}^{\text {up }}$ the diagonal matrices collecting the mass coefficients of the nodes belonging to the lower and upper surfaces, respectively.

Using in (12) the linear transformation (11), the system motion can be expressed in terms of corner nodal and corner fibers dofs as follows:

$$
\delta \widehat{\mathbf{a}}_{e}^{T} \widehat{\mathbf{M}}_{e} \widehat{\mathbf{a}}_{e}=\delta \widehat{\mathbf{a}}_{e}^{T} \widehat{\mathbf{f}}_{e},
$$

with

$$
\begin{aligned}
\underset{24 \times 24}{\widehat{\mathbf{M}}_{e}} & =\mathbf{Q}^{T} \mathbf{M}_{e} \mathbf{Q}=\left[\begin{array}{c}
\left(\mathbf{m}^{u p}+\mathbf{m}^{l o w}\right) \\
\left(\mathbf{m}^{u p}-\mathbf{m}^{\text {low }}\right)\left(\mathbf{m}^{\text {low }}\right) \\
\left(\mathbf{m}^{u p}+\mathbf{m}^{l o w}\right)
\end{array}\right]_{e}, \\
\widehat{\mathbf{f}}_{e} & =\mathbf{Q}^{T} \mathbf{f}_{e}=\left[\begin{array}{c}
\mathbf{f}_{1-4}+\mathbf{f}_{5-8} \\
-\mathbf{f}_{1-4}+\mathbf{f}_{5-8}
\end{array}\right]_{e} .
\end{aligned}
$$

As discussed in Cocchetti et al (2015), the off-diagonal terms of $\widehat{\mathbf{M}}_{e}$ are zero for parallelepiped elements and are small compared to the diagonal ones for acceptably 
distorted elements, so that they can be safely set to zero. In the following, the lumped transformed mass matrix $\widehat{\mathbf{M}}_{e_{\text {lump }}}$ will be considered:

$$
\widehat{\mathbf{M}}_{e_{l u m p}}=\left[\begin{array}{cc}
\left(\mathbf{m}^{u p}+\mathbf{m}^{\text {low }}\right) & \mathbf{0} \\
\mathbf{0} & \left(\mathbf{m}^{u p}+\mathbf{m}^{\text {low }}\right)
\end{array}\right]_{e} .
$$

In structural dynamics problems, the structural response is mainly determined by individual elements translational rigid body modes, which are governed by corner nodes dofs. From this consideration, it follows that the element maximum frequency can be reduced by increasing only the mass coefficients related to the corner fibers dofs, which concern relative displacements and rotations between the upper and lower surfaces and are therefore related to higher eigenmodes and eigenfrequencies. In this way, the lowest eigenfrequencies are left mainly unaffected and the structural response is well reproduced. To this purpose, let the scaled transformed mass matrix $\widehat{\mathbf{M}}_{e_{\text {lump }}^{\alpha}}^{\alpha}$ be defined as:

$$
\widehat{\mathbf{M}}_{e_{\text {lump }}}^{\alpha}=\left[\begin{array}{cc}
\left(\mathbf{m}^{u p}+\mathbf{m}^{l o w}\right) & \mathbf{0} \\
\mathbf{0} & \alpha_{e}\left(\mathbf{m}^{u p}+\mathbf{m}^{\text {low }}\right)
\end{array}\right]_{e},
$$

where $\alpha_{e}$ is the element mass scaling parameter, so that equation (14) becomes:

$$
\delta \widehat{\mathbf{a}}_{e}^{T} \widehat{\mathbf{M}}_{e_{l u m p}}^{\alpha} \widehat{\mathbf{a}}_{e}=\delta \widehat{\mathbf{a}}_{e}^{T} \widehat{\mathbf{f}}_{e} .
$$

The selective mass scaling in (18) leaves the inertia associated to the element translational rigid body modes unaltered, while the inertia associated to rotational rigid body modes is increased. The effect of this spurious increase has been discussed in Cocchetti et al (2015) and it has been shown to be negligible in most cases. However, one must be aware that this type of mass scaling could not be suitable for problems where the rotational rigid body component of the motion is prevailing.

When a single-layer problem is addressed, the selective mass scaling technique proposed in Cocchetti et al $(2013,2015)$ has been shown to preserve the diagonal structure not only of a single element mass matrix, but also of the assembled structure, when its motion is described as in (19) in terms of transformed dofs. This implies that transformed element mass contributions in (18), coming from adjacent elements sharing the same global dofs, have to be assembled. This is straightforward for single layer shells, since their inertia and force terms can be easily summed up, as they depend only on the dofs pertaining to the considered corner fiber.

\section{Selective mass scaling of multiple layers shells}

When a layered structure is considered, several solid-shell elements, one for each layer is assumed hereafter, are used for the discretization through the thickness (Fig. 2). In this case, the term "fiber" defines the multi-layer segment connecting all the nodes through the shell thickness at the same in-plane position. As shown in Fig. 2, a multi-layer fiber is then formed by a set of corner fibers belonging to the elements stacked up along the thickness. Unlike in the case of a single layer shell, the procedure described in Section 2 cannot be directly applied, since corner fiber 


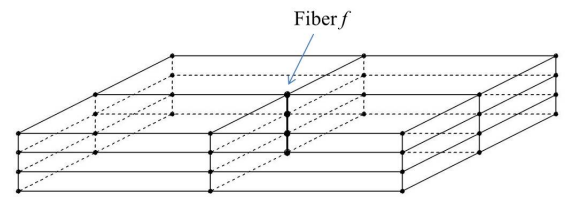

Fig. 2 Fiber in a multi-layered shell structure

dofs cannot be assembled through the thickness. This implies that the momentum balance has to be enforced in terms of the original displacement dofs.

After applying the mass scaling, the virtual work equation can be expressed back in terms of the original nodal dofs, by introducing the inverse transformation $(8)_{2}$ into equation (19):

$$
\delta \mathbf{a}_{e}^{T} \underbrace{\mathbf{Q} \frac{1}{2} \widehat{\mathbf{M}}_{e_{l u m p}}^{\alpha} \frac{1}{2} \mathbf{Q}^{T}}_{\mathbf{M}_{e}^{\alpha}} \mathbf{a}_{e}=\delta \mathbf{a}_{e}^{T} \underbrace{\mathbf{Q} \frac{1}{2} \widehat{\mathbf{f}}_{e}}_{\mathbf{f}_{e}} .
$$

The scaled mass matrix of a generic solid-shell element, belonging to the layer $l$ of the laminate, expressed in terms of the original dofs, takes the form:

$$
\mathbf{M}_{e}^{\alpha}=\frac{1}{4}\left(\mathbf{Q} \widehat{\mathbf{M}}_{e_{l u m p}^{\alpha}}^{\alpha} \mathbf{Q}^{T}\right)=\frac{1}{4}\left[\begin{array}{l}
\left(1+\alpha_{e}\right)\left(\mathbf{m}^{l o w}+\mathbf{m}^{u p}\right)\left(1-\alpha_{e}\right)\left(\mathbf{m}^{l o w}+\mathbf{m}^{u p}\right) \\
\left(1-\alpha_{e}\right)\left(\mathbf{m}^{l o w}+\mathbf{m}^{u p}\right)\left(1+\alpha_{e}\right)\left(\mathbf{m}^{l o w}+\mathbf{m}^{u p}\right)
\end{array}\right] .
$$

Let us focus on a single fiber $f$ and define the mass matrix $\mathbf{M}_{l f}^{\alpha}$ of one of its layers $l$ as the assembly of the mass contributions from the elements sharing that fiber (4 elements in Fig. 2) and belonging to the same layer $l$ :

$$
\underset{24 \times 24}{\mathbf{M}_{l f}^{\alpha}}=\underset{e}{\mathrm{~A}_{e}}\left[\mathbf{M}_{e}^{\alpha}\right]_{l f}=\frac{1}{4}\left[\begin{array}{ll}
\mathbf{m}_{l}^{L L} & \mathbf{m}_{l}^{L U} \\
\mathbf{m}_{l}^{U L} & \mathbf{m}_{l}^{U U}
\end{array}\right]_{f},
$$

where $\mathrm{A}$ is the assembly operator, and superscripts $U$ and $L$ refer to the upper and lower nodes contributions, respectively, of layer $l$ :

$$
\begin{aligned}
& \mathbf{m}_{12 \times 12}^{L L}=\underset{12 \times 12}{\mathbf{m}_{l}^{U U}}=\underset{e}{\mathrm{~A}}\left(1+\alpha_{e}\right)\left(\mathbf{m}_{e}^{u p}+\mathbf{m}_{e}^{\text {low }}\right)_{l}, \\
& \mathbf{m}_{12 \times 12}^{L U}=\mathbf{m}_{12 \times 12}^{U L}=\underset{e}{\mathbf{A}}\left(1-\alpha_{e}\right)\left(\mathbf{m}_{e}^{u p}+\mathbf{m}_{e}^{\text {low }}\right)_{l} .
\end{aligned}
$$

The overall solution can be expressed in the form of decoupled fiber subproblems, each of them in the form

$$
\mathbf{M}_{f}^{\alpha} \mathbf{a}_{f}=\mathrm{A}_{l} \mathbf{M}_{l f}^{\alpha} \mathbf{a}_{f}=\mathbf{f}_{f}
$$

where $\mathbf{M}_{f}^{\alpha}$ is the scaled mass matrix of fiber $f$ obtained assembling the layer mass matrices through the thickness and $\mathbf{f}_{f}$ refers to the equivalent nodal forces for the nodes along that fiber only. For each fiber $f$, the fiber mass matrix $\mathbf{M}_{f}^{\alpha}$ has a tridiagonal structure, with $3 \times\left(N_{l}+1\right)$ rows, $N_{l}$ being the number of layers. This can be easily seen considering the three-layer example of Fig. $3\left(N_{l}=3\right)$, where 


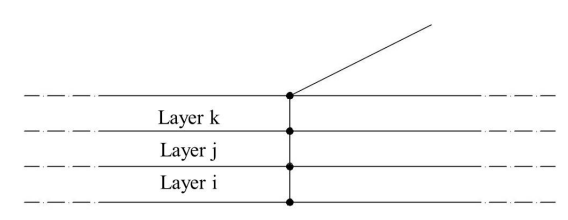

Fig. 3 Example of three-layered fiber

the mass matrix $\mathbf{M}_{f}^{\alpha}$ related to the fiber $f$ crossing the layers $i, j$, and $k$ is given by:

$$
\underset{12 N_{l} \times 12 N_{l}}{\mathbf{M}_{f}^{\alpha}}=\left[\begin{array}{cccc}
\mathbf{m}_{i}^{L L} & \mathbf{m}_{i}^{L U} & \mathbf{0} & \mathbf{0} \\
\mathbf{m}_{i}^{U L} & \mathbf{m}_{i}^{U U}+\mathbf{m}_{j}^{L L} & \mathbf{m}_{j}^{L U} & \mathbf{0} \\
\mathbf{0} & \mathbf{m}_{j}^{U L} & \mathbf{m}_{j}^{U U}+\mathbf{m}_{k}^{L L} & \mathbf{m}_{k}^{L U} \\
\mathbf{0} & \mathbf{0} & \mathbf{m}_{k}^{U L} & \mathbf{m}_{k}^{U U}
\end{array}\right] .
$$

The overall solving system can be obtained by assembling over all the fibers, namely:

$$
\mathbf{M}^{\alpha} \mathbf{a}=\mathrm{A}_{f} \mathbf{M}_{f} \mathbf{a}_{f}=\mathrm{A}_{f} \mathbf{f}_{f}=\mathbf{f}
$$

where the global mass matrix $\mathbf{M}^{\alpha}$ is a block diagonal matrix, each block consisting of a tridiagonal matrix of the type in (25), corresponding to the dofs of a single fiber $f$. In view of the mild tridiagonal coupling, the computation of the accelerations at fiber nodes can be carried out very effectively, e.g. by an explicit $L U$ decomposition of the fiber mass matrix, involving a number of dofs proportional to the layer number, which is usually small. The small burden, additional with respect to the case of a fully diagonal mass matrix, is by far compensated by the larger stable time step which can be used in the computation.

\section{Optimal mass scaling factor and maximum eigenfrequency computation}

The definition of the optimal value of the selective mass scaling factor $\alpha$ is the result of a trade-off between two conflicting objectives. On one side, large values of $\alpha$ lead to an increasing reduction of the element maximum eigenfrequency, allowing for the use of larger stable time steps. On the other side, an accurate reproduction of the structural dynamical properties requires to consider values of $\alpha$ as small as possible. For a deformed solid-shell element, a typical reduction of the maximum eigenfrequency with the mass scaling factor is shown in Fig. 4 (Cocchetti et al (2015)). While for small values of $\alpha$ there is a rapid gain in terms of time step size, for large values of $\alpha$ there is almost no gain at the cost of a growing loss of accuracy in the structural response, due to the modified mass. It is therefore essential to define a criterion for the definition of the optimal element scaling factor.

In unstructured meshes, the elements are all different and $\alpha$ has to be computed individually for each element in a pre-processing step. After defining the element optimal scaling factor, one has to compute the time step size to be used for stable integration with the central difference scheme. In large deformation problems, the element size and shape can change considerably during the analysis, possibly 


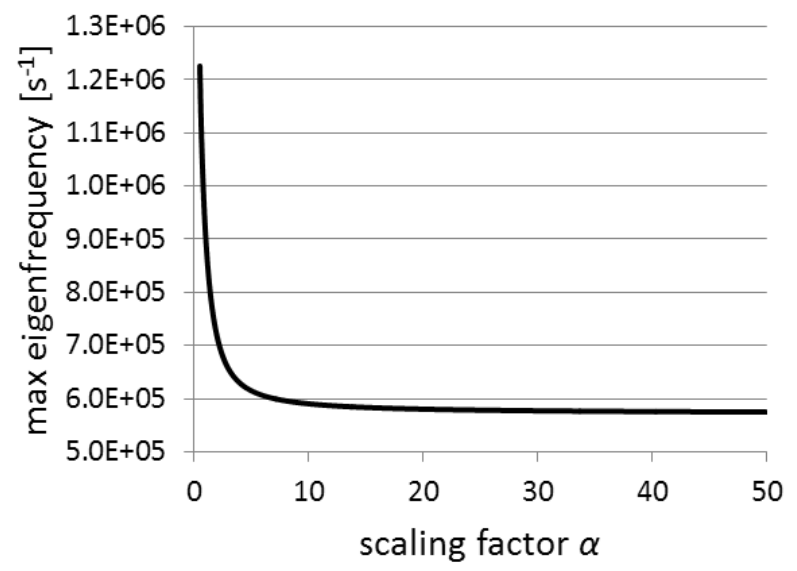

Fig. 4 Decrease of $\max$ eigenfrequency $\omega_{\max }$ for increasing scaling factor $\alpha$.

requiring a repeated run-time computation of the critical time step. A computationally effective technique for the estimation of the critical time step is therefore also necessary.

Since it can be shown that the maximum element eigenfrequency is associated to the thickness vibration mode, in Cocchetti et al (2015) an equivalence has been established between selective and geometric scaling, where geometric scaling means artificially increasing the element thickness while the in-plane dimensions are kept constant. Interpreting the Jacobian $\mathbf{J}=\partial \mathbf{x} / \partial \boldsymbol{\xi}$ of the isogeometric mapping as the deformation gradient of the deformation process transforming the parent cubic $2 \times 2 \times 2$ element into the current distorted shape, $\mathbf{c}=\mathbf{J}^{-T} \mathbf{J}^{-1}$ is the Cauchy deformation tensor and

$$
\mathbf{c}=\sum_{i=1}^{3} \gamma_{i}^{2} \mathbf{t}_{i} \otimes \mathbf{t}_{i}
$$

represents its spectral decomposition, where $\gamma_{1}^{2} \leq \gamma_{2}^{2} \leq \gamma_{3}^{2}$ are the principal stretches and $\mathbf{t}_{3}$ represents the direction of maximum shortening in the current configuration, i.e. the thickness direction of the distorted element.

The procedure proposed in Cocchetti et al (2015) consists of defining

$$
\alpha^{o p t}=\left(\frac{\gamma_{3}}{\gamma_{2}}\right)^{2}
$$

so that after the scaling the thickness becomes of a size comparable to the element in-plane dimensions. Alternatively, if $L_{1}, L_{2}, h_{0}$ define the approximate element in-plane and thickness dimensions as the distances between centroids of element faces, i.e.

$$
\begin{aligned}
L_{1} & =\|\mathbf{x}(1,0,0)-\mathbf{x}(-1,0,0)\|, L_{2}=\|\mathbf{x}(0,1,0)-\mathbf{x}(0,-1,0)\|, \\
h_{0} & =\|\mathbf{x}(0,0,1)-\mathbf{x}(0,0,-1)\|,
\end{aligned}
$$


a quick and inexpensive estimate of $\alpha^{\text {opt }}$ can be achieved by setting

$$
\alpha_{\text {opt }}=\frac{L_{\min }^{2}}{h_{0}^{2}}, \quad L_{\min }=\min \left\{L_{1}, L_{2}\right\} .
$$

For parallelepiped elements, the criteria in (28) and (30) are equivalent.

In the present context of laminated shells, where several solid-shell elements are stacked one on top of the other, the layers have in general different thicknesses and a different value of $\alpha^{\text {opt }}$ has to be computed for each layer, according to either (28) or (30). As it will be shown in the numerical examples, the selective mass scaling will allow to use the same time step, only dictated by the element in-plane dimensions, no matter what is the number of layers used to discretize the shell thickness.

Once $\alpha$ has been chosen for each element in the mesh, the size of the stable time step has to be approximated rigorously from below, to avoid an unstable time integration. The stable time step is determined according to (1) and (2), where $\omega_{\max }^{e}$ is estimated for each element according to the procedure proposed in Cocchetti et al (2015) for arbitrarily distorted solid-shell elements with scaled masses, which is based on the procedure proposed by Flanagan and Belytschko (1984) for constant strain hexahedra (for simplicity of notation the element index $e$ is hereafter discarded). An upper bound $\omega_{G}^{2}$ of $\omega_{\max }$ is first computed making use of Gershgorin's theorem as:

$$
\omega_{\text {max }}^{2} \leq \omega_{G}^{2}=\frac{2 \mu}{\rho_{0}}\left[\frac{\nu}{1-2 \nu} I_{1}^{1 / \alpha}+\max _{i}\left\{\sum_{j=1}^{3}\left|\left[\mathbf{c}_{0}^{1 / \alpha}\right]_{i j}\right|\right\} .\right.
$$

In (31), $\mu$ is the elastic shear modulus, $\nu$ is Poisson's ratio, $\rho$ is the mass density, $\mathbf{c}_{0}^{1 / \alpha}$ is the Cauchy deformation tensor of the scaled element, defined as:

$$
\mathbf{c}_{0}^{1 / \alpha}=\left(\mathbf{J}_{0}^{\sqrt{\alpha}}\right)^{-T}\left(\mathbf{J}_{0}^{\sqrt{\alpha}}\right)^{-1}, \quad \mathbf{J}_{0}^{\sqrt{\alpha}}=\mathbf{J}_{0} \mathbf{I}_{3}^{\sqrt{\alpha}}, \quad \mathbf{I}_{3}^{\sqrt{\alpha}}=\left[\begin{array}{ccc}
1 & 0 & 0 \\
0 & 1 & 0 \\
0 & 0 & \sqrt{\alpha}
\end{array}\right],
$$

where $\mathbf{J}_{0}$ is the Jacobian of the element isoparametric mapping evaluated at the element centroid. $I_{1}^{1 / \alpha}$ in (31) is the first invariant of $\mathbf{c}_{0}^{1 / \alpha}$.

A sharper rigorous bound $\omega_{N-R}^{2}$ on $\omega_{\max }^{e}$ can be obtained for each element performing one Newton-Raphson iteration for the search of the maximum root of the characteristic equation $f\left(\omega^{2} ; \alpha\right)=0$ of the element eigenvalue problem, starting from the tentative solution $\omega^{2}=\omega_{G}^{2}$ :

$$
\omega_{N-R}^{2}=\omega_{G}^{2}-\frac{f\left(\omega_{G}^{2} ; \alpha^{o p t}\right)}{f^{\prime}\left(\omega_{G}^{2} ; \alpha^{o p t}\right)}
$$

where

$$
\begin{aligned}
f\left(\omega^{2} ; \alpha\right)= & \left(\omega^{2}\right)^{3}-\frac{1-\nu}{1-2 \nu} \frac{2 \mu}{\rho_{0}} I_{1}^{1 / \alpha}\left(\omega^{2}\right)^{2} \\
& +\frac{1}{1-2 \nu}\left(\frac{2 \mu}{\rho_{0}}\right)^{2} I_{2}^{1 / \alpha} \omega^{2}-\frac{1+\nu}{1-2 \nu}\left(\frac{2 \mu}{\rho_{0}}\right)^{3} I_{3}^{1 / \alpha}=0 \\
f^{\prime}\left(\omega^{2} ; \alpha\right)= & \frac{d f}{d \omega^{2}}
\end{aligned}
$$




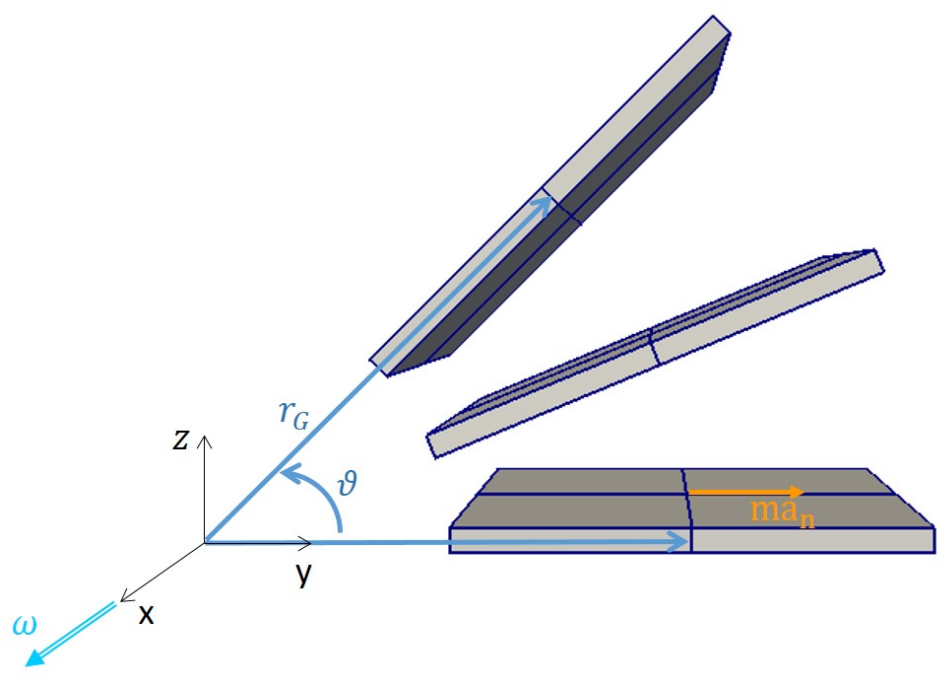

Fig. 5 Rotational patch test: geometry.

and $I_{2}^{1 / \alpha}, I_{3}^{1 / \alpha}$ are the second and third invariants, respectively, of $\mathbf{c}_{0}^{1 / \alpha}$.

As discussed in Cocchetti et al (2015) on the basis of an extensive numerical investigation, $\omega_{N-R}^{2}$ approximates $\omega_{\max }$ from above with an average approximation of about $1 \%$ and a peak error of about $3 \%$.

\section{Numerical results}

Seven numerical examples are here proposed to check the accuracy of the mass scaling procedure and the computational gain. The Q1ST solid-shell element, developed in Schwarze and Reese (2011), is used in all cases and the scaling parameter is computed using either the simplified approach in (30) or the procedure in (28).

\subsection{Rotational patch test}

As highlighted in Section 2, the proposed selective mass scaling technique affects rigid body rotations. The rotational patch test presented in Cocchetti et al (2015) is here considered to numerically asses the effect of selective mass scaling on the rotational inertia in the presence of layered shells. The parallelepiped patch, shown in Fig. 5, has a square in-plane shape of size $L=2 \mathrm{~m}$ and thickness $H=0.1 \mathrm{~m}$. A uniform angular velocity $\omega=\frac{\pi}{4} \frac{\mathrm{rad}}{\mathrm{s}}$ around the $x$ axis is imposed to the boundary nodes. Furthermore, in order to reproduce the rigid body rotation conditions, centripetal body forces of the same magnitude of the theoretical centrifugal inertia forces, but with opposite sign, are applied to the elements of the patch. The introduction of the selectively scaled mass matrix causes a deviation from the situation of perfectly rigid body motion. In Cocchetti et al (2015), it has been proposed 
Table 1 Rotational patch test: comparison between scaled and unscaled time step sizes.

\begin{tabular}{cccccc}
\hline Number of layers & $\begin{array}{c}\text { Layer thickness } \\
(\mathrm{m})\end{array}$ & $\gamma$ & $\alpha$ & $\begin{array}{c}\Delta t^{\alpha} \\
(\mu \mathrm{s})\end{array}$ & $\begin{array}{c}\Delta t^{0} \\
(\mu \mathrm{s})\end{array}$ \\
\hline 1 & 0.100 & 20 & 100 & 126.49 & 17.21 \\
2 & 0.050 & 40 & 400 & 126.49 & 8.62 \\
4 & 0.025 & 80 & 1600 & 126.49 & 4.31 \\
\hline
\end{tabular}

to evaluate the error $\epsilon$ due to the modified rotational inertia in terms of kinetic energy:

$$
\epsilon=\frac{K^{\alpha}-K^{0}}{K^{0}}
$$

where $K^{\alpha}$ and $K^{0}$ are the kinetic energies of the scaled and of the original system, respectively. The energy error for the patch has been shown to be well approximated by the following expression, correlating the error to the mass scaling factor, to the element geometry and to the distance $R_{G}$ between the patch centroid and the rotation axis:

$$
\epsilon=(\alpha-1) \frac{1}{4 \gamma^{2}\left(\frac{R_{G}}{L}\right)^{2}},
$$

being $\gamma=\frac{L}{h}$ the ratio between the patch width and the element thickness. This expression shows that the error decreases with the square of the ratio $R_{G} / L$, which measures the importance of the translational component of the motion with respect to the rotational one.

The analysis has been performed considering increasing values of the distance $R_{G}$, namely $R_{G}=[2,4,6,8,10,20]$. Moreover, the simulations have been run considering a plate discretization with 1,2 and 4 homogeneous layers of equal thickness. Four square elements are used for the in-plane discretization. Table 1 gives the values of the mass scaling factor $\alpha$, equal for all elements in the mesh, of the ratio $\gamma$ in these three cases and of the stable time steps $\Delta t^{\alpha}$ and $\Delta t^{0}$ computed with scaled and unscaled masses, respectively. The computational gain can be easily evaluated by comparing the time steps.

Figure 6 shows the trend of the energy error (35) for increasing values of the ratio between the radius $R_{G}$ and the in-plane patch size $L$. The numerical results are consistent with the analytical estimate in (36): the numerical error decreases almost quadratically at increasing distance $R_{G}$. It can also be observed that the numerical solutions obtained for the three cases are, as expected, almost perfectly overlapped, despite the different number of layers and the fact that the same time step has been used in all cases.

\subsection{Small displacements cantilever beam}

In this second example the cantilever beam depicted in Fig. 7 is considered to show the capability of the proposed selective mass scaling procedure to reproduce the dynamical behavior of a linear elastic thin structure in small displacements, over a time interval spanning several fundamental periods of the beam. The beam 


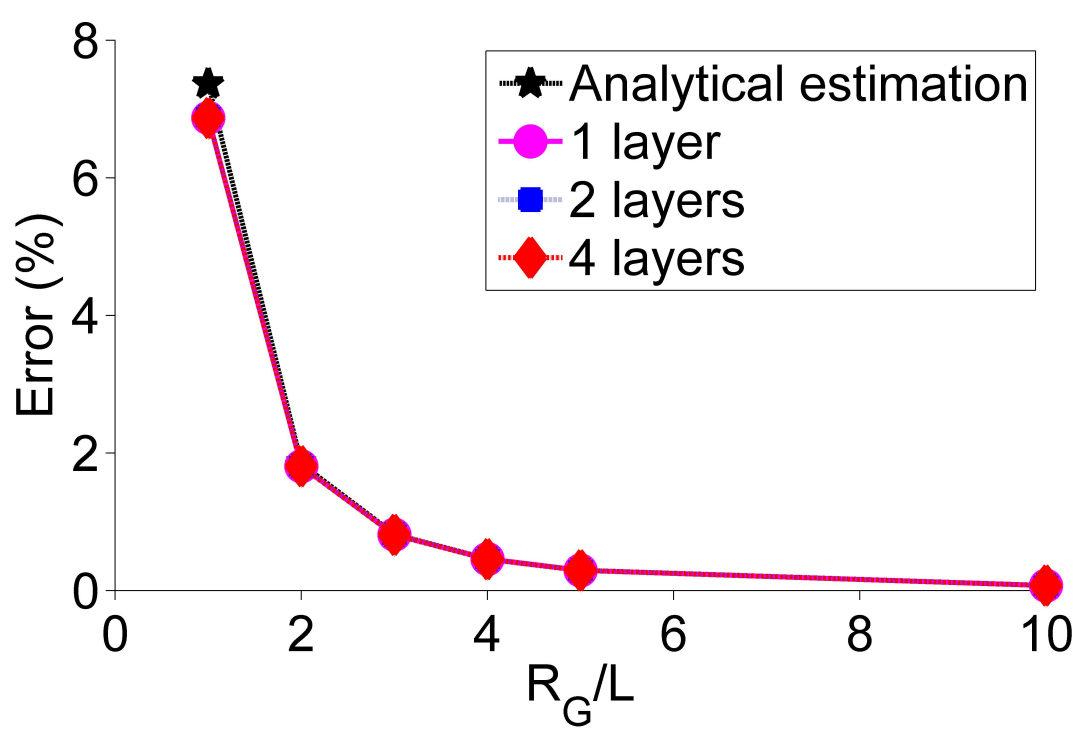

Fig. 6 Rotational patch test: comparison between numerical and analytical error.

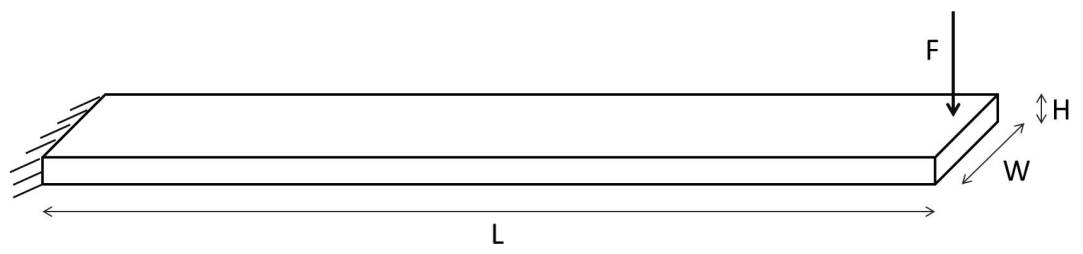

Fig. 7 Small displacements cantilever beam: geometry, boundary and loading conditions.

has a length $L=6000 \mathrm{~mm}$ and a rectangular cross section width $W=200 \mathrm{~mm}$ and depth $H=100 \mathrm{~mm}$. A material with Young's modulus $E=2 \times 10^{5} \mathrm{MPa}$, Poisson's ratio $\nu=0.3$ and density $\rho=7500 \mathrm{~kg} / \mathrm{m}^{3}$ has been chosen. The analysis has been performed considering an increasing number of homogeneous layers of equal thickness, namely $1,2,4,6,8,10$. In all cases, the cantilever is clamped at one side and a concentrated step load $F=500 \mathrm{~N}$, which remains constant in time, is applied at the free tip, equally distributed among the tip nodes. A small load has been chosen, so that small displacements can be assumed, with tip displacement over beam length ratio of the order of $10^{-3}$ in the static case.

Two different discretizations, displayed in Fig. 8, have been considered, denoted in the following as mesh $\mathrm{A}$ and mesh $\mathrm{B}$, respectively: the first mesh is made of six regular parallelepiped elements, while six distorted elements have been used for the second one. For mesh A, the application of equations (28) and (30) for the computation of $\alpha$ gives the same result:

$$
\alpha=N_{l}^{2} \frac{W^{2}}{H^{2}}=4 N_{l}^{2}
$$




\begin{tabular}{|l|l|l|l|l|l|}
\hline 1 & 2 & 3 & 4 & 5 & 6 \\
\hline
\end{tabular}

a) regular elements mesh

\begin{tabular}{|l|l|l|l|l|l|}
\hline 1 & 2 & 3 & 4 & 5 & 6 \\
\hline
\end{tabular}

b) distorted elements mesh

Fig. 8 Small displacements cantilever beam: top view of adopted meshes. a) Mesh A, regular elements. b) Mesh B, distorted elements.

Table 2 Small displacements cantilever beam: mass scaling factors for mesh A.

\begin{tabular}{ccc}
\hline Number of layers & $\begin{array}{c}\text { Layer thickness } \\
(\mathrm{mm})\end{array}$ & $\alpha$ \\
\hline 1 & 100.00 & 4.00 \\
2 & 50.00 & 16.00 \\
4 & 25.00 & 64.00 \\
6 & 16.67 & 144.00 \\
8 & 12.50 & 256.00 \\
10 & 10.00 & 400.00 \\
\hline
\end{tabular}

Table 3 Small displacements cantilever beam: mass scaling factors for mesh B computed according to (28).

\begin{tabular}{ccccc}
\hline Element number & 1 layer & 2 layers & 4 layers & 6 layers \\
\hline 1 & 3.98 & 15.93 & 63.73 & 143.39 \\
2 & 4.00 & 15.98 & 63.94 & 143.86 \\
3 & 3.89 & 15.55 & 62.20 & 139.96 \\
4 & 3.98 & 15.92 & 63.70 & 143.33 \\
5 & 3.96 & 15.83 & 63.32 & 142.49 \\
6 & 3.99 & 15.98 & 63.91 & 143.79 \\
\hline
\end{tabular}

Table 4 Small displacements cantilever beam: mass scaling factors for mesh B computed according to (30).

\begin{tabular}{cccccc}
\hline $\begin{array}{c}\text { Element } \\
\text { number }\end{array}$ & 1 layer & 2 layers & 4 layers & 6 layers & 8 layers \\
\hline 1 & 4.31 & 17.23 & 68.93 & 151.37 & 275.71 \\
2 & 4.12 & 16.46 & 65.85 & 148.10 & 262.40 \\
3 & 5.70 & 22.81 & 91.25 & 205.23 & 364.99 \\
4 & 4.35 & 17.39 & 69.57 & 156.47 & 278.28 \\
5 & 4.99 & 19.96 & 79.84 & 179.57 & 319.36 \\
6 & 4.25 & 16.98 & 67.92 & 152.76 & 271.68 \\
\hline
\end{tabular}

and the scaling factor increases with the square of the layers number $N_{l}$. The obtained values are shown in Table 2 . For mesh B, since we intentionally gave to the elements different distortions, a different scaling factor is obtained for each element. The values obtained with equation (28) and (30) are shown in Tables 3 and 4. Also in this case, the scaling factor increases with the square of $N_{l}$ and similar values are obtained with the two methods. 
Table 5 Small displacements cantilever beam: updated mass scaling factors for mesh B computed according to $(28)$.

\begin{tabular}{ccccccc}
\hline Element number & 1 layer & 2 layers & 4 layers & 6 layers & 8 layers & 10 layers \\
\hline 1 & 3.78 & 15.12 & 60.46 & 136.04 & 241.85 & 377.88 \\
2 & 3.74 & 14.96 & 59.84 & 134.63 & 239.34 & 373.97 \\
3 & 3.89 & 15.55 & 62.20 & 139.96 & 248.81 & 388.77 \\
4 & 3.78 & 15.10 & 60.40 & 135.90 & 241.59 & 377.49 \\
5 & 3.78 & 15.13 & 60.53 & 136.20 & 242.14 & 378.34 \\
6 & 3.73 & 14.93 & 59.70 & 134.33 & 238.80 & 373.13 \\
\hline
\end{tabular}

Table 6 Small displacements cantilever beam: number of layers and critical time step before mass scaling.

\begin{tabular}{cccc}
\hline Number of layers & $\begin{array}{c}\text { Layer thickness } \\
(\mathrm{mm})\end{array}$ & $\begin{array}{c}\Delta t^{0}(\mu \mathrm{s}) \\
\text { mesh A }\end{array}$ & $\begin{array}{c}\Delta t^{0}(\mu \mathrm{s}) \\
\text { mesh B }\end{array}$ \\
\hline 1 & 100.00 & 16.21 & 16.17 \\
2 & 50.00 & 8.29 & 8.29 \\
4 & 25.00 & 4.17 & 4.17 \\
6 & 16.67 & 2.78 & 2.78 \\
8 & 12.50 & 2.09 & 2.08 \\
10 & 10.00 & 1.67 & 1.67 \\
\hline
\end{tabular}

Table 7 Small displacements cantilever beam: critical time step after mass scaling.

\begin{tabular}{ccc}
\hline $\begin{array}{c}\Delta t^{\alpha}(\mu \mathrm{s}) \\
\text { mesh A }\end{array}$ & $\begin{array}{c}\Delta t^{\alpha}(\mu \mathrm{s}) \\
\text { mesh B - } \alpha \text { eq. }(28)\end{array}$ & $\begin{array}{c}\Delta t^{\alpha}(\mu \mathrm{s}) \\
\text { mesh B }-\alpha \text { eq. (30) }\end{array}$ \\
\hline 27.83 & 27.65 & 28.00 \\
\hline
\end{tabular}

Since the elements are different, according to (2) the critical time step to be used for time integration is determined by the element exhibiting the highest eigenfrequency $\bar{\omega}_{\max }=\max \left\{\omega_{\max }^{e}\right\}$. A possible intervention, aimed at minimizing the alteration of the structural dynamical properties, consists of reducing all the scale factors in Tables 3 and 4, with the exception of the one of the element with the maximum eigenfrequency $\bar{\omega}_{\max }$, in such a way that all scaled elements possess the same highest eigenfrequency. This can be easily done by setting $\omega^{2}=\bar{\omega}_{\text {max }}^{2}$ in $(34)_{1}$ and then solving for $\alpha$ for each element. This additional computation has to be carried out only once, in a pre-processing step, and therefore is not expensive.

The recomputed values of $\alpha$ in Table 3 are shown in Table 5. For the distorted mesh $\mathrm{B}$, the critical element is the number 3 and its scaling factor remains unchanged. With the exception of element 3, all scaling factors are smaller than those in Table 3. The differences between values in the two tables are not large, since the elements have similar sizes. Much larger differences can be expected in strongly unstructured meshes.

Table 6 shows the stable time steps $\Delta t^{0}$ for the unscaled mass matrix, computed for the two meshes for increasing number of layers: it can be noted that the time step size decreases linearly with the layers number. The time step sizes obtained after application of mass scaling, using the values of $\alpha$ in Tables 3 and 4 are shown in Table 7. For both meshes, the obtained value of $\Delta t^{\alpha}$, determined by the in-plane 


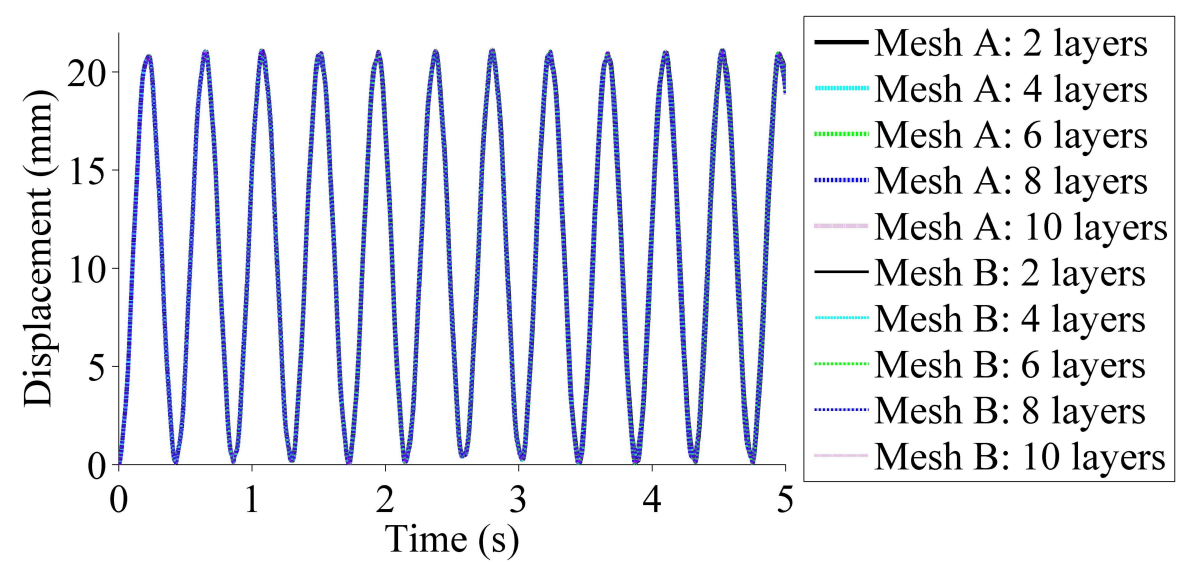

Fig. 9 Small displacements cantilever beam: time history of tip displacement.

element size, is independent of the number of layers. Therefore, only one value is shown in the table. In the 10 layers case, the computational gain, quantified as the ratio between the adopted time step and $\Delta t^{0}$, is of about 16.7 times for mesh $\mathrm{B}$ and slightly higher for mesh A.

The time evolution of the vertical tip displacement is displayed in Fig. 9: it can be seen that the curves, obtained with a different number of layers, are almost perfectly overlapped, even when multiple time periods are considered. A zoom on the first time period for the 10-layer case is shown in Fig. 10 to get a refined view on the differences between the numerical solutions. Note that the analytical static solution for the tip displacement is equal to $10.8 \mathrm{~mm}$.

\subsection{Cantilever beam: large displacements case}

The large displacements dynamical response of the impulsively loaded cantilever beam shown in Fig. 11 is analyzed. The beam length is equal to $100 \mathrm{~mm}$, while its cross section is $20 \mathrm{~mm}$ wide and $2 \mathrm{~mm}$ deep. A linear elastic material has been considered with $E=1768 \mathrm{MPa}, \nu=0.3$ and $\rho=3000 \mathrm{~kg} / \mathrm{m}^{3}$. A uniform transverse tip load per unit surface $p$, linearly increasing with time from 0 to $0.5 \cdot 10^{-3} \mathrm{MPa}$ at $t=5 \mathrm{~ms}$, is applied and distributed over the tip nodes. The analysis is run for $5 \mathrm{~ms}$, after which very large tip displacements are achieved. An increasing number of homogeneous layers of equal thickness has been considered, namely 1,2,4,8,16. Table 8 provides the values of the mass scaling factor, computed according to eq. 28 , and of the stable time step. As in the previous example, it can be noted that the critical time step is not affected by the reduction in the element thickness, but it is determined only by the in-plane size. The comparison between the time histories of vertical tip displacements obtained in the different cases is displayed in Fig. 12.

Displaced configurations for varying number of layers, at the final time instant $t=5 \mathrm{~ms}$, are shown in Fig. 13. Despite the high values of $\alpha=1600$ used in the 16 layers case and the notably large rotations of some elements, there are no visible differences in the displaced configurations in the four cases shown in Fig. 13. 


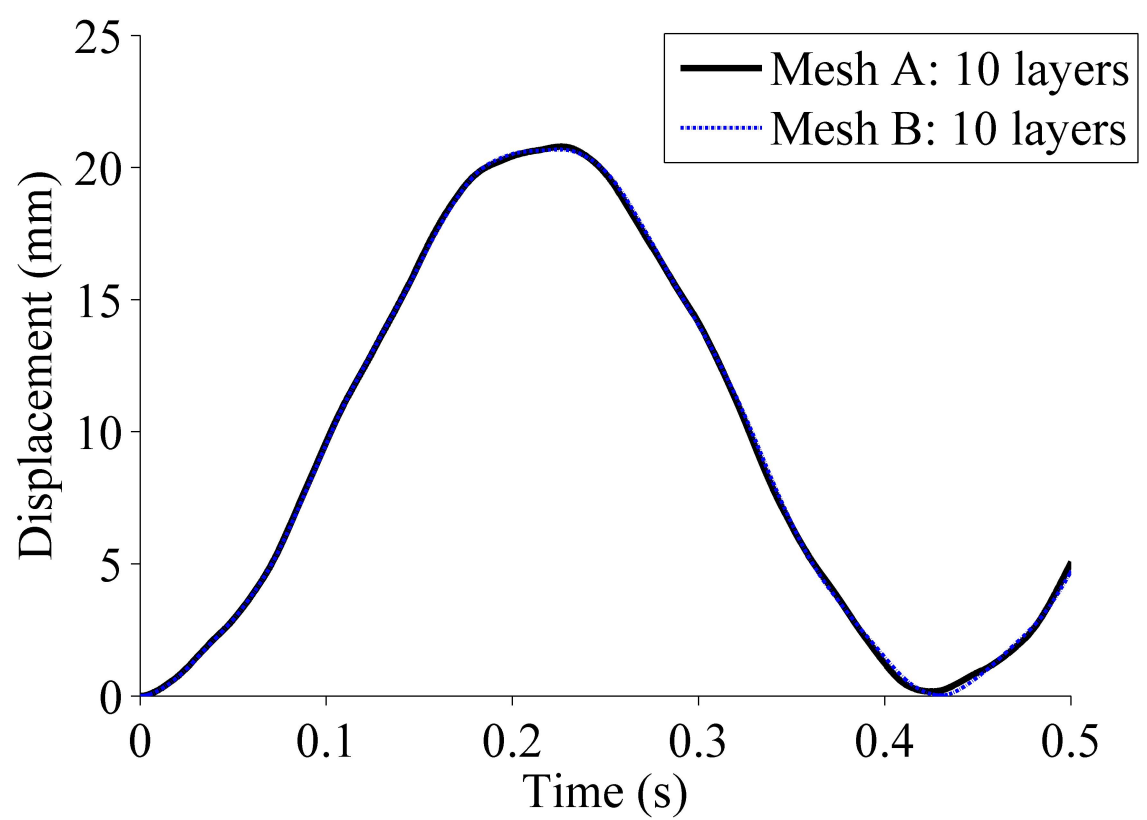

Fig. 10 Small displacements cantilever beam. Tip vertical displacement: zoom on the first time period.

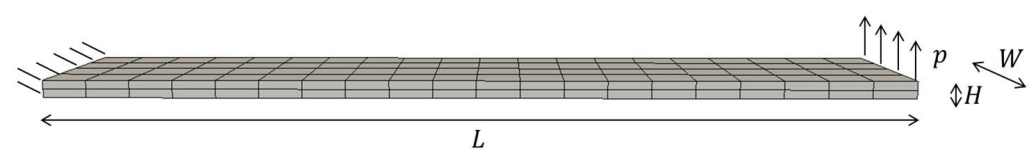

Fig. 11 Large displacements cantilever beam: geometry, boundary and loading conditions.

Table 8 Large displacements cantilever beam: mass scaling factor and critical time step size.

\begin{tabular}{ccccc}
\hline Number of layers & $\begin{array}{c}\text { Layer thickness } \\
(\mathrm{mm})\end{array}$ & $\alpha$ (eq. 28) & $\begin{array}{c}\Delta t^{\alpha} \\
(\mu \mathrm{s})\end{array}$ & $\begin{array}{c}\Delta t^{0} \\
(\mu \mathrm{s})\end{array}$ \\
\hline 1 & 2.00 & 6.25 & 4.119 & 2.171 \\
2 & 1.00 & 25.00 & 4.119 & 1.114 \\
4 & 0.50 & 100.00 & 4.119 & 0.560 \\
8 & 0.25 & 400.00 & 4.119 & 0.281 \\
16 & 0.125 & 1600.00 & 4.119 & 0.140 \\
\hline
\end{tabular}

\subsection{Pinched cylinder}

A cylindrical shell, clamped at one end, is loaded at the other end by a pair of concentrated forces applied at two diametrically opposite points, as shown in Fig. 14. This benchmark problem has been proposed in Ibrahimbegovic et al (2001). Because of symmetry, only one half of the specimen is considered. The length of the cylinder is $L=3.048 \mathrm{~m}$, while its radius is equal to $R=1.016 \mathrm{~m}$. The 


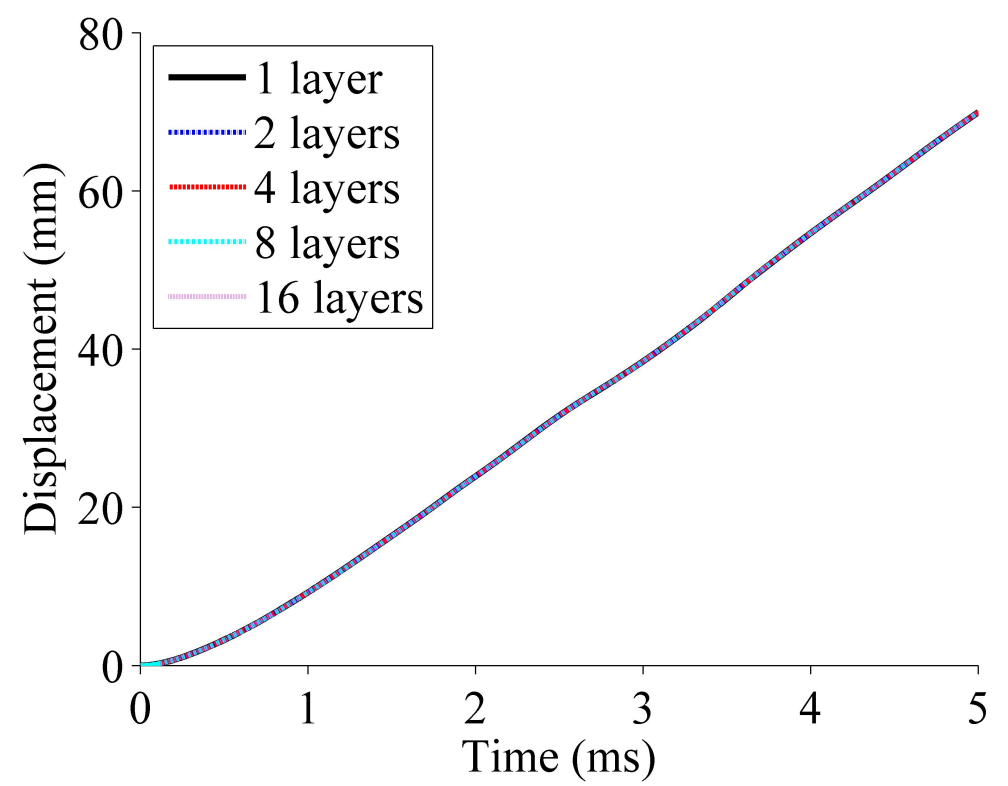

Fig. 12 Large displacements cantilever beam: time history of vertical tip displacement.

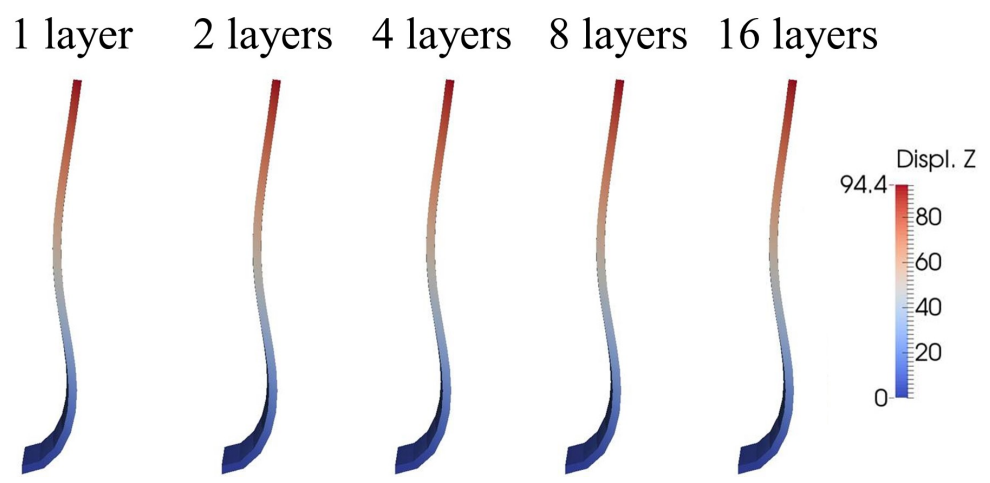

Fig. 13 Large displacements cantilever beam: deformed shapes at $t=5 \mathrm{~ms}$.

thickness is equal to $t=0.03 \mathrm{~m}$. The material has been considered linear elastic with Young's modulus $E=2.0685 \cdot 10^{7} \mathrm{~N} / \mathrm{m}^{2}$, Poisson's ratio $\nu=0.3$ and density $\rho=1000 \mathrm{~kg} / \mathrm{m}^{3}$. The concentrated load increases linearly with time from $0 \mathrm{~N}$ to $900 \mathrm{~N}$ in $0.9 \mathrm{~s}$.

The simulation has been performed considering three different numbers $N_{l}$ of homogeneous layers of uniform thickness, namely 2, 4 and $6.1920 \times N_{l}$ solid-shell elements have been used in the discretization. Table 9 provides the values of the time step and of the maximum mass scaling factor determined in the three cases on the basis of the simplified procedure in (30). Thanks to the mass scaling, the same time step is obtained in all cases. The same analysis has also been run with the commercial code Abaqus using the same number of classical S4R shell elements, to 


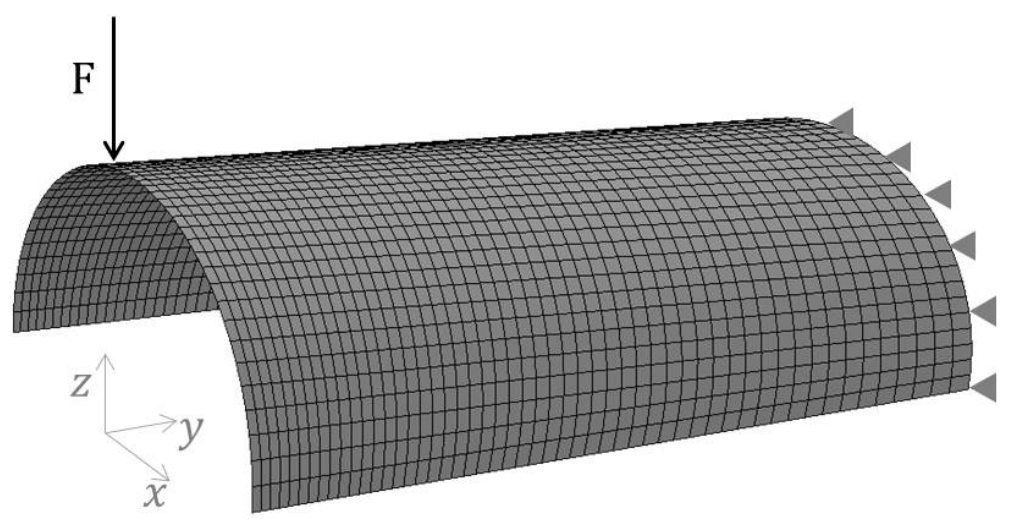

Fig. 14 Pinched cylinder: geometry, mesh, boundary and loading conditions.

Table 9 Pinched cylinder: stable time step and mass scaling factor

\begin{tabular}{ccc}
\hline Number of layers & $\begin{array}{c}\Delta t^{\alpha} \\
(\mathrm{ms})\end{array}$ & $\alpha$ eq. (30) \\
\hline 2 & 0.1504 & 26.81 \\
4 & 0.1504 & 107.60 \\
6 & 0.1504 & 242.63 \\
\hline
\end{tabular}

test the numerical results obtained with the implemented code. The time histories of the displacement along $z$ of the loading point are displayed in Fig. 15. It can be noted that the four curves are almost perfectly overlapped up to the end of the analysis. In Fig. 16 the contour plots of the displacement along $z$ obtained in the three cases are compared to the result of the Abaqus simulation. A very good agreement can be observed also in this case.

\subsection{Peeling test}

A double cantilever beam subjected to peeling-like loading conditions is simulated in this example with the aim to assess the modifications of the throughthe-thickness stresses due to the mass scaling application. Figure 17 shows the analyzed geometry overlapped to the final deformed shape. The left end of the beam is clamped, while a vertical displacement, linearly increasing in time from 0 to $5 \mathrm{~mm}$ in $0.1 \mathrm{~s}$, is prescribed on the right end of the specimen, at the tip of the free arm. By exploiting symmetry, only the upper half of the beam of total thickness of $10 \mathrm{~mm}$ (i.e. the peeling arm thickness is equal to $5 \mathrm{~mm}$ ) is analyzed. The beam is $24 \mathrm{~mm}$ wide and has a total length of $200 \mathrm{~mm}$ with a peeling arm of $100 \mathrm{~mm}$. The peeling arm has been discretized by means of five layers of equal thickness. The beam is made of steel with Young's modulus $E=210000 \mathrm{MPa}$, Poisson's ratio $\nu=0.2$ and mass density $\rho=7800 \mathrm{~kg} / \mathrm{m}^{3}$. Since the mesh is structured and all the layers have the same thickness, a unique value of mass scaling 


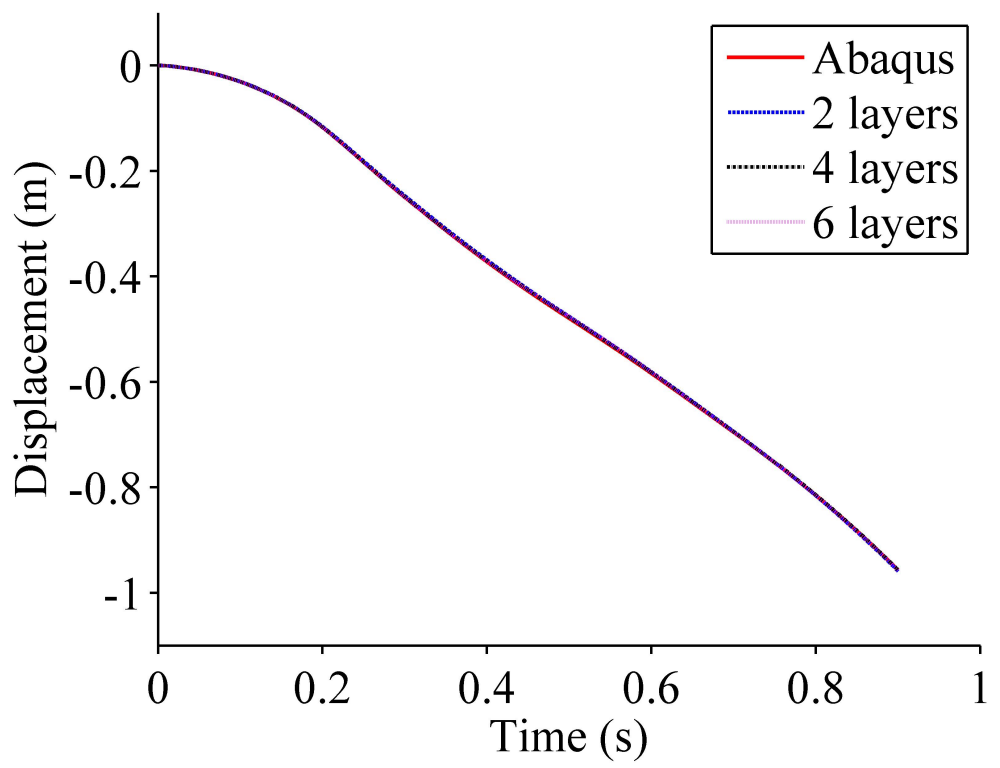

Fig. 15 Pinched cylinder: displacement along $z$ of the loaded point.
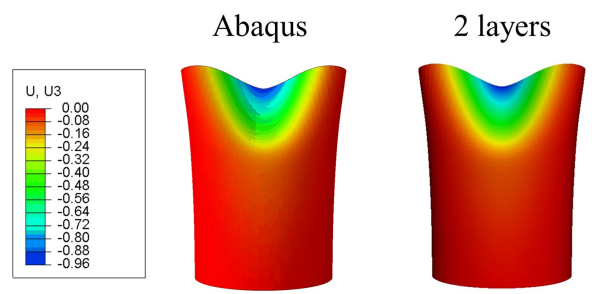

4 layers

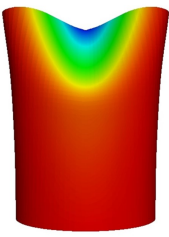

6 layers

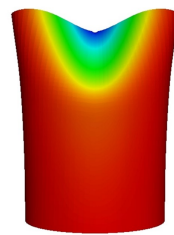

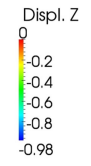

Fig. 16 Pinched cylinder: contour plots of displacement along $z$.

parameter has been computed, $\alpha=59.17$. The critical time steps, evaluated applying the mass scaling procedure or not, are equal to $\Delta t^{0}=1.8264 \cdot 10^{-6} \mathrm{~s}$ and $\Delta t^{\alpha}=1.6201 \cdot 10^{-5} \mathrm{~s}$, respectively.

The distribution of the stress component $\sigma_{x}$ along the beam thickness, being direction $x$ aligned with the beam axis, has been evaluated at the centroids of the five elements B-C-D-E-F placed at the right of the beam mid-length, i.e. the first elements of the peeling arm (see Fig. 17), at five subsequent time instants (Fig. 18). The two stress distributions appear to be almost identical throughout the thickness.

Figure 19 shows the time evolution of the $\sigma_{z}$ stress component, being $z$ the thickness direction, evaluated at the centroid of element A, placed at the left of the beam mid-length at the bottom of the cross section (Fig. 17), with and without selective mass scaling. In both cases, the representative stress points have been plotted at about every $10^{-3} \mathrm{~s}$, and oscillate with comparable amplitude around the 


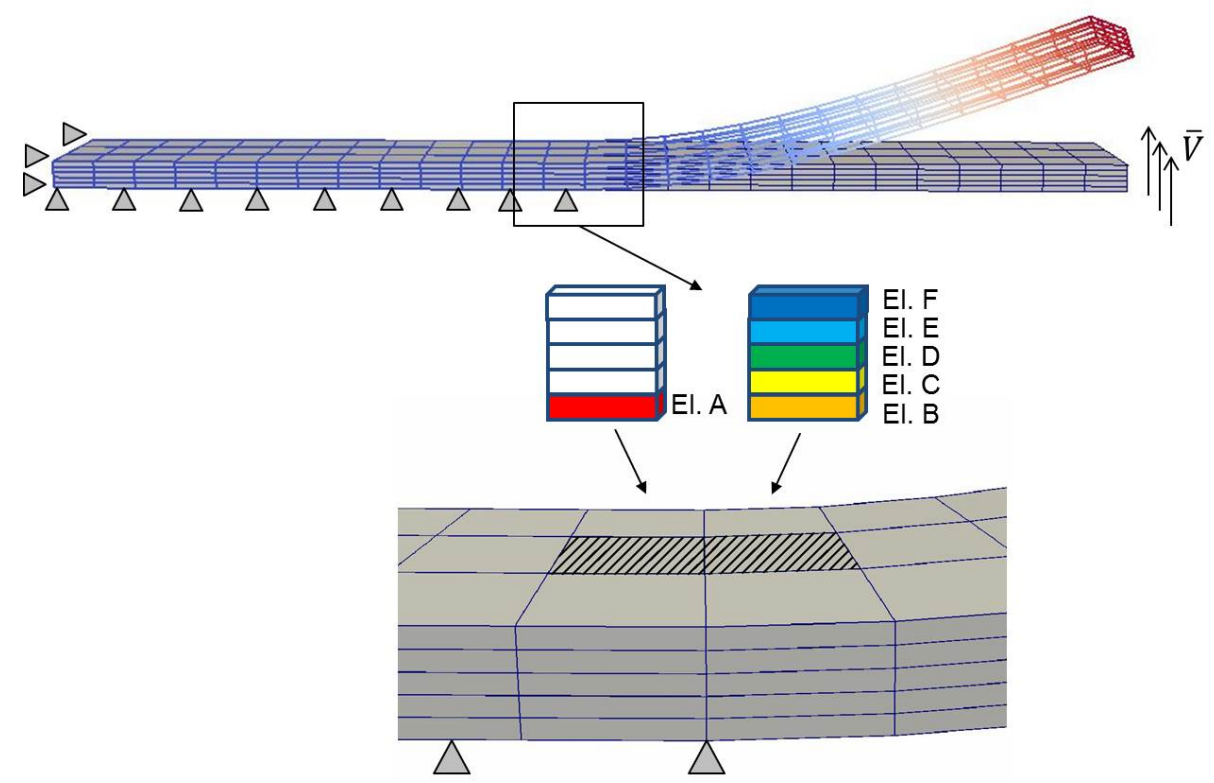

Fig. 17 Peeling test: geometry, boundary conditions, discretization and final deformed shape.

same straight line defining the stress growth due to the linearly growing intensity of the applied load.

\subsection{Sandwich beam with soft core}

While structures with uniform material through the thickness were considered in the previous examples, in this case the bending response of a cantilever sandwich beam with soft core is addressed. The beam length is equal to $600 \mathrm{~mm}$, while its rectangular cross section is $60 \mathrm{~mm}$ wide and $20 \mathrm{~mm}$ deep. As shown in Fig. 20 , one side of the beam is completely clamped, while a surface load, linearly increasing with time from 0 to $0.05 \mathrm{MPa}$ in $0.04 \mathrm{~s}$, is applied at the opposite one. As in Sokolinsky et al (2003), the sandwich beam is composed of two external aluminum thin face sheets and of a soft core of low-density PVC foam. Both aluminum layers are $0.5 \mathrm{~mm}$ thick, while the thickness of the soft core is equal to $19 \mathrm{~mm}$. The mechanical properties of the two materials are listed in Table 10. Each aluminum layer has been discretized by only one solid-shell element throughthe-thickness, while five solid-shell elements of equal thickness are stacked up to model the soft core. The adopted mesh, characterized by 2520 solid-shell elements, has been obtained considering an in-plane size of the elements equal to $10 \mathrm{~mm}$. Although the mesh is structured, two different values of the mass scaling parameter have to be defined, since different materials and element thicknesses are present: in particular, the mass matrices of the elements belonging to the aluminum layers are scaled by $\alpha=400$, while $\alpha=6.93$ is adopted for the elements belonging to 


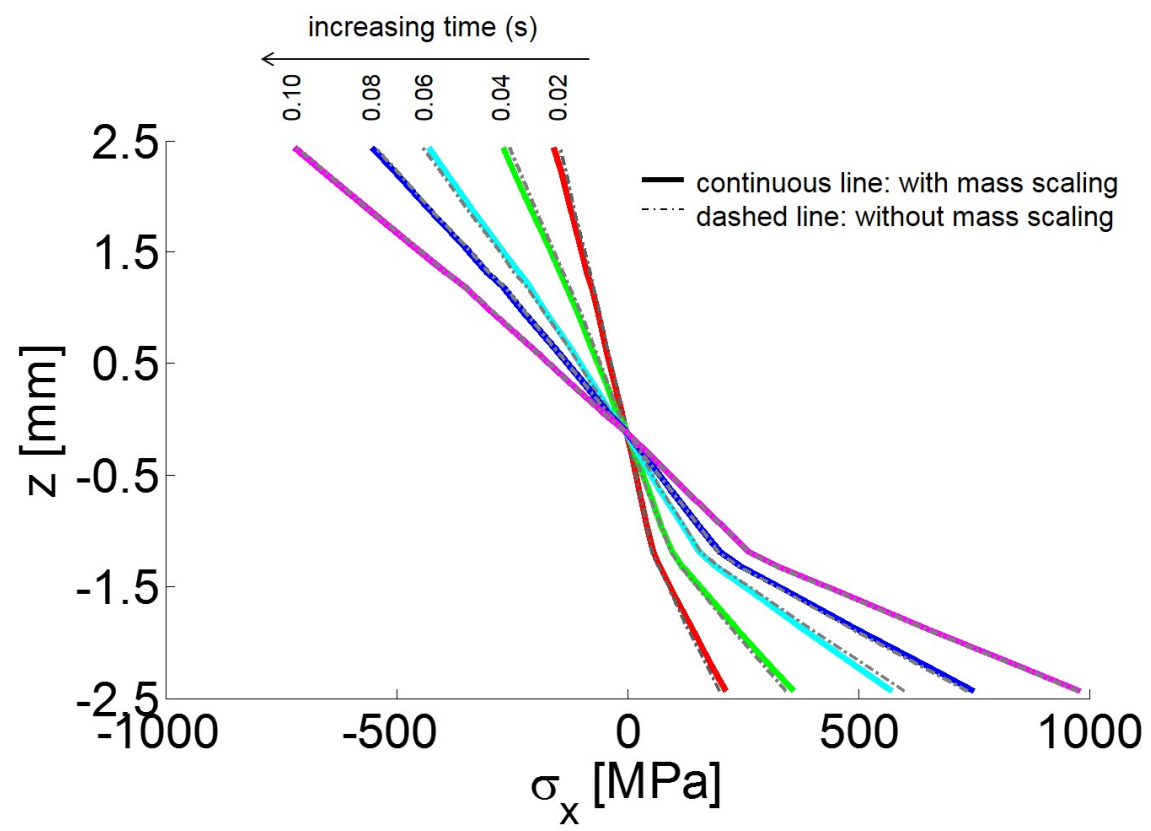

Fig. 18 Peeling test: longitudinal stress component $\sigma_{x}$ at the elements B-C-D-E-F (see Fig. 17) of the free arm.

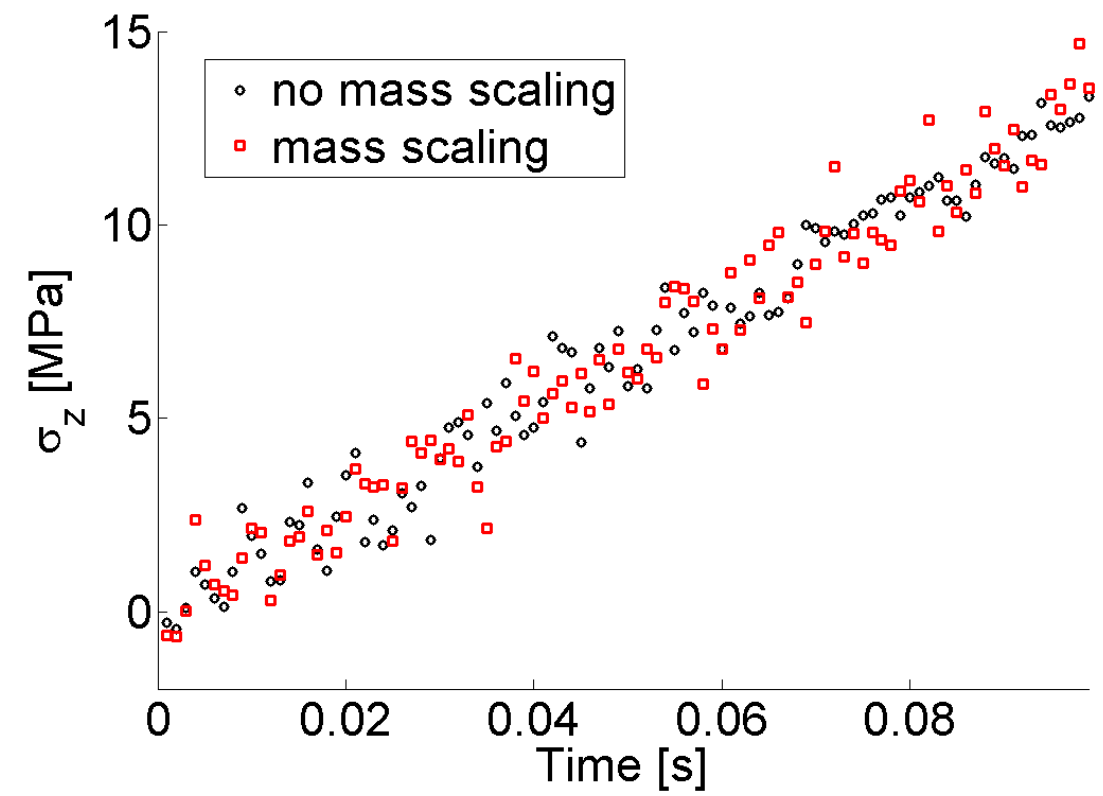

Fig. 19 Peeling test: vertical stress component $\sigma_{z}$ at element A (see Fig. 17) of the clamped region. 


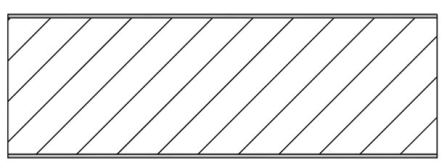

Aluminum

PVC foam

Aluminum

Fig. 20 Sandwich beam with soft core: geometry, material layers and boundary conditions.

Table 10 Sandwich beam with soft core: material properties

\begin{tabular}{cccc}
\hline Material & $\begin{array}{c}\text { Density } \\
\left(\frac{\mathrm{kg}}{\mathrm{m}^{3}}\right)\end{array}$ & $\begin{array}{c}\text { Young's modulus } \\
(\mathrm{MPa})\end{array}$ & Poisson's ratio \\
\hline Aluminum (0.5 mm-thick) & 2,700 & 72,400 & 0.33 \\
Low density PVC foam & 60 & 58 & 0.32 \\
\hline
\end{tabular}

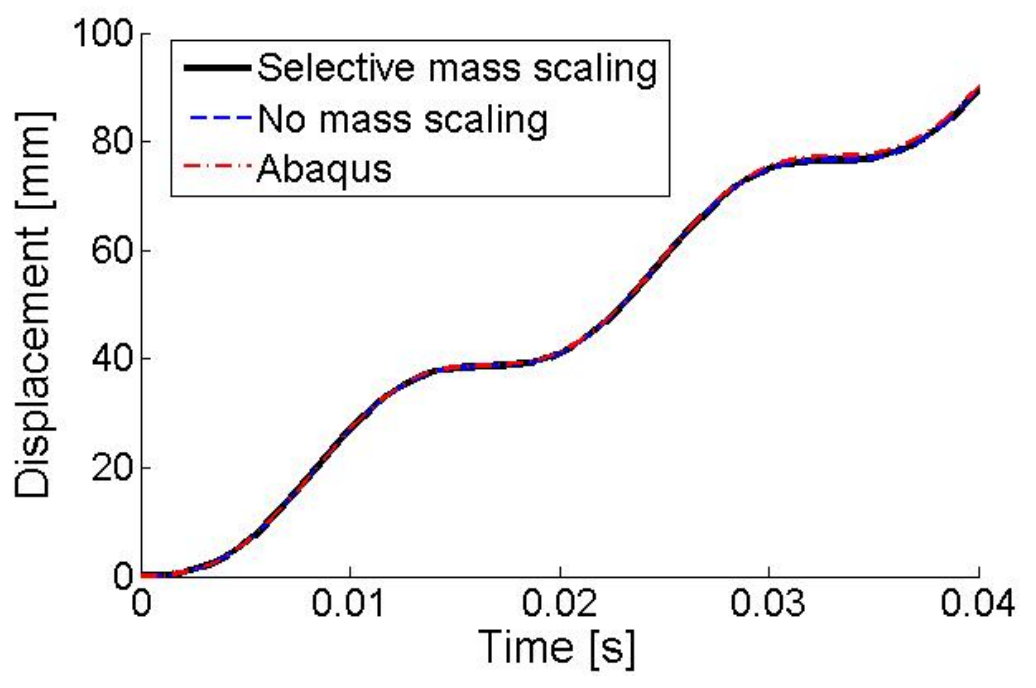

Fig. 21 Sandwich beam with soft core: vertical tip displacement.

the soft core. The stable time step is equal to $1.126 \mu \mathrm{s}$ or to $0.079 \mu \mathrm{s}$, depending on whether the selective mass scaling technique is applied or not.

In order to test the numerical results obtained with the implemented code, the analysis has also been run with the commercial code Abaqus, using S4R shell elements with composite section and the same in-plane size of the solid-shell discretization. In Figure 21, the vertical displacement of the loaded end of the beam computed with the proposed technique is compared both with the numerical re- 


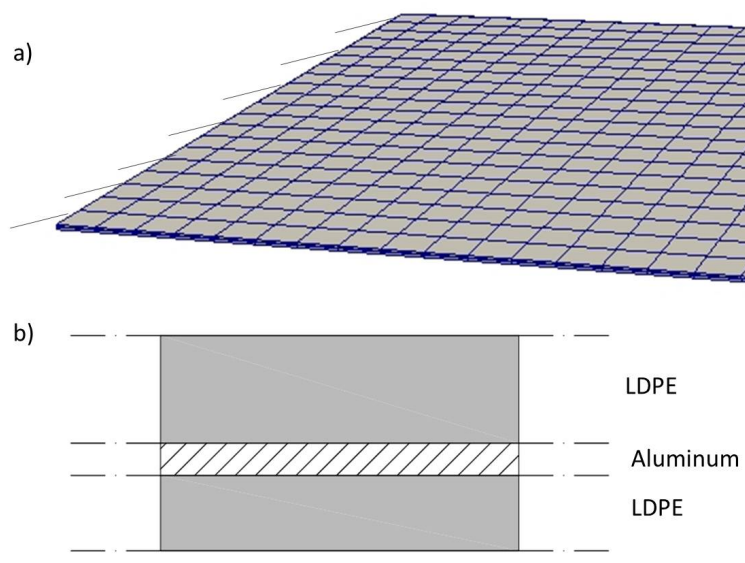

Fig. 22 Sandwich plate with hard core: a) geometry and boundary conditions, b) material layers.

sult obtained without applying the selective mass scaling procedure and with those obtained using the commercial code.

\subsection{Sandwich plate with hard core}

A $10 \mathrm{~mm} \times 10 \mathrm{~mm}$ square sandwich plate with hard core, fully clamped at one edge, is subject to a transverse distributed load, applied at the opposite one and linearly increasing with time from 0 to $0.0025 \mathrm{MPa}$ in $0.5 \mathrm{~ms}$ (Fig. 22a). The plate is composed of two external layers of low density polyethylene (LDPE) with thicknesses equal to $30 \mu \mathrm{m}$ (upper layer) and $21 \mu \mathrm{m}$ (lower layer), and of an internal layer of aluminum of thickness $9 \mu \mathrm{m}$, as shown in Fig. 22b. The overall thickness of the cross section is equal to $60 \mu \mathrm{m}$. The material properties are listed in Table 5.4 and are taken from Frangi et al (2010). Because of the small thicknesses involved in the model, the through-the-thickness discretization has been obtained using one solid-shell element per layer. The in-plane size of the element is equal to $0.5 \mathrm{~mm}$ : the resulting mesh of 1200 elements is shown in Fig. 22 together with the boundary conditions of the problem. Three different values of the mass scaling parameters have been computed: the mass matrices of the elements belonging to the three layers (bottom up) are scaled by $\alpha$ equal to $277.78,3086.42$, and 566.89 , respectively. The stable time steps, computed by applying or not the selective mass scaling, are equal to $9.487 \cdot 10^{-8} \mathrm{~s}$ and $2.327 \cdot 10^{-9} \mathrm{~s}$, respectively. The elements belonging to the aluminum layer turn out to be the critical ones in determining the maximum eigenfrequency $\bar{\omega}_{\max }$. The mass scaling parameters of the other elements are not re-computed after the estimation of $\bar{\omega}_{\max }$ in order to consider a more severe test on the accuracy of the numerical results. The loaded edge deflection is plotted in Fig. 23. The maximum displacement reached at the end of the analysis is about 
Table 11 Sandwich plate with hard core: material properties

\begin{tabular}{cccc}
\hline Material & $\begin{array}{c}\text { Density } \\
\left(\frac{\mathrm{kg}}{\mathrm{m}^{3}}\right)\end{array}$ & $\begin{array}{c}\text { Young's modulus } \\
(\mathrm{MPa})\end{array}$ & Poisson's ratio \\
\hline Aluminum $(9 \mu$ m-thick $)$ & 2,700 & 30,000 & 0.30 \\
Low density polyethylene (LDPE) & 1,000 & 500 & 0.40 \\
\hline
\end{tabular}

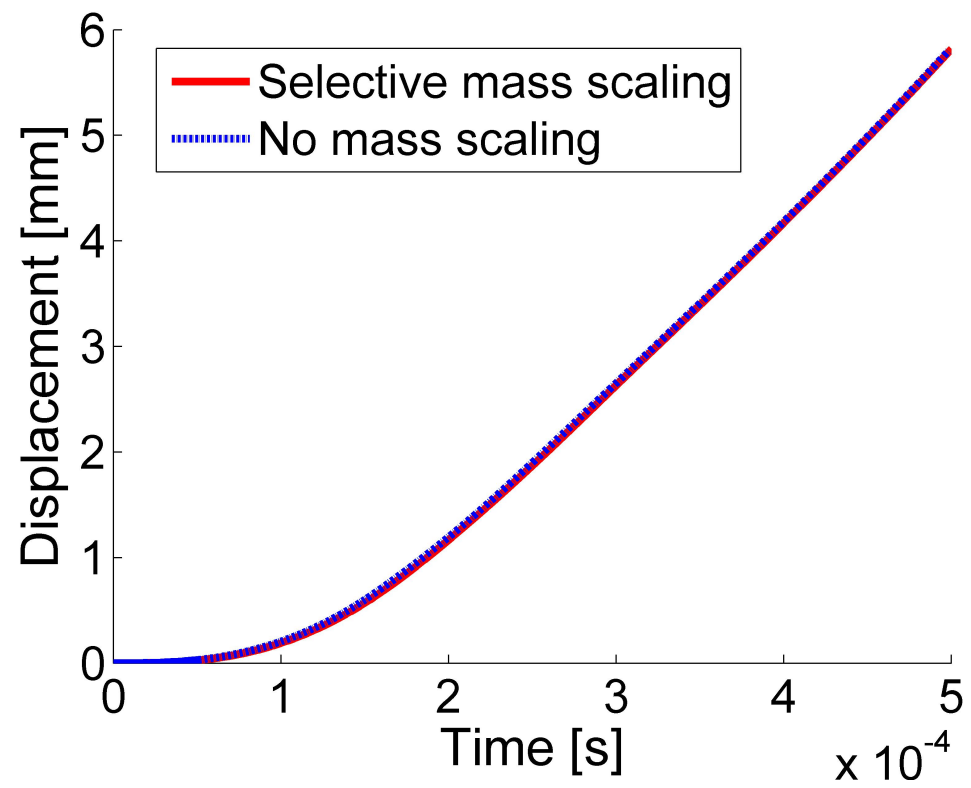

Fig. 23 Sandwich plate with hard core: maximum vertical displacement.

one hundred times greater than the plate thickness.

\section{Conclusions}

A selective mass scaling procedure for layered thin-walled structures, discretized with one or more solid-shell elements per layer, has been presented and its performance has been assessed by means of several numerical examples. The method is a natural extension to multi-layer structures of what has been proposed in Cocchetti et al (2013); Pagani et al (2014); Cocchetti et al (2015) for homogeneous, onelayer shells. The scaling procedure can be applied to distorted 8-node solid-shell elements, with nodal displacements dofs. The proposed scaling reduces the highest eigenfrequencies, while the lower ones, associated to the rigid body translations, remain almost unaffected. As a result, when the dynamical behavior is governed by the lowest frequencies, the structural response is well reproduced. The inertia associated to rotational rigid body motions is increased by the mass scaling. This implies a modification of the resulting kinetic energy that, however, is shown to be 
negligible when the translational component of the motion of individual elements is dominant.

The mass matrix resulting from the application of the selective mass scaling is block diagonal, where each block corresponds to the degrees of freedom of a single through-the-thickness fiber, and it is characterized by a tridiagonal structure. Its dimensions are directly related to the number of layers, typically small. Therefore, the solution of the small linear system providing the accelerations of the nodes belonging to the same fiber is inexpensive and the small additional burden is by far compensated by the largest stable time step.

A critical role is played by the choice of the mass scaling factor. The criterion proposed in Cocchetti et al (2015) has been used together with the strategy proposed there for the computation of the critical time step size. The considered numerical tests have shown that, using this criterion, it is possible to perform explicit dynamics simulations with an increasing number of solid-shell elements through the shell thickness, using the same stable time step, independent of the number of layers and determined by the in-plane element size, with negligible accuracy loss.

Acknowledgements The financial support by Tetra Pak Packaging Solutions is kindly acknowledged.

\section{References}

Abed-Meraim F, Combescure A (2009) An improved assumed strain solid-shell element formulation with physical stabilization for geometric non-linear applications and elastic-plastic stability analysis. International Journal for Numerical Methods in Engineering 80(13):1640-1686

Abed-Meraim F, T VD, Combescure A (2013) New quadratic solid-shell elements and their evaluation on linear benchmark problems. Computing 95(5):373-394

Askes H, Nguyen DCD, Tyas A (2011) Increasing the critical time step: microinertia, inertia penalties and mass scaling. Computational Mechanics 47(6):657667

Cocchetti G, Pagani M, Perego U (2013) Selective mass scaling and critical timestep estimate for explicit dynamics analyses with solid-shell elements. Computers \& Structures 127:39-52

Cocchetti G, Pagani M, Perego U (2015) Selective mass scaling for distorted solid-shell elements in explicit dynamics: optimal scaling factor and stable time step estimate. International Journal for Numerical Methods in Engineering 101(9):700-731

Flanagan D, Belytschko T (1984) Eigenvalues and stable time steps for the uniform strain hexaedron and quadilateral. Journal of Applied Mechanics 51(1):35-40

Frangi A, Pagani M, Perego U, Borsari R (2010) Directional cohesive elements for the simulation of blade cutting of thin shells. Computer Modeling in Engineering and Sciences (CMES) 57(3):205

Hauptmann R, Schweizerhof K (1998) A systematic development of 'solid-shell' element formulations for linear and non-linear analyses employing only displacement degrees of freedom. International Journal for Numerical Methods in Engineering 42(1):49-69 
Hetherington J, Rodriguez-Ferran A, Askes H (2012) A new bipenalty formulation for ensuring time step stability in time domain computational dynamics. International Journal for Numerical Methods in Engineering 90:269-286

Ibrahimbegovic A, Brank B, Courtois P (2001) Stress resultant geometrically exact form of classical shell model and vector-like parametrization of constrained finite rotations. International Journal for Numerical Methods in Engineering 52:12351252

Macek RW, Aubert BH (1995) A mass penalty technique to control the critical time increment in explicit dynamic finite element analyses. Earthquake Engineering and Structural Dynamics 24(10):1315-1331

Meyers MA (1994) Dynamic behavior of materials. Wiley, New York

Naceur H, Shiri S, Coutellier D, Batoz JL (2013) On the modeling and design of composite multilayered structures using solid-shell finite element model. Finite Elements in Analysis and Design 70-71:1-14

Olovsson L, Unosson M, Simonsson K (2004) Selective mass scaling for thin walled structures modeled with tri-linear solid elements. Computational Mechanics $34(2): 134-136$

Olovsson L, Simonsson K, Unosson M (2005) Selective mass scaling for explicit finite element analyses. International Journal for Numerical Methods in Engineering 63(10):1436-1445

Pagani M, Reese S, Perego U (2014) Computationally efficient explicit nonlinear analyses using reduced integration-based solid-shell finite elements. Computer Methods in Applied Mechanics and Engineering 268:141-159

Schwarze M, Reese S (2011) A reduced integration solid-shell finite element based on the EAS and the ANS concept: large deformation problems. International Journal for Numerical Methods in Engineering 85(3):289-329

Sokolinsky VS, Shen H, Vaikhanski L, Nutt SR (2003) Experimental and analytical study of nonlinear bending response of sandwich beams. Composite Structures 60:219-229

Tan X, Vu-Quoc L (2005) Efficient and accurate multilayer solid-shell element: non-linear materials at finite strain. International Journal 63(15):2124-2170

Tkachuk A, Bischoff M (2013a) Local and global strategies for optimal selective mass scaling. Computational Mechanics 53(6):1197-1207

Tkachuk A, Bischoff M (2013b) Variational methods for selective mass scaling. Computational Mechanics 52(3):563-570

Tkachuk A, Bischoff M (2015) Direct and sparse construction of consistent inverse mass matrices: general variational formulation and application to selective mass scaling. International Journal for Numerical Methods in Engineering 101(6):435469

Zukas JA (2004) Introduction to hydrocodes. Elsevier, Amsterdam 